\title{
Supramolecular Effects in Dendritic Systems Containing Photoactive Groups
}

\author{
GIANLUCA CAMILLO AZZELLINI \\ Instituto de Química, Departamento de Química Fundamental, USP, \\ Cx. Postal 26077 - 05513-970 São Paulo, SP, Brazil
}

Manuscript received on September 8, 1999; accepted for publication on September 15, 1999; presented by José M. RIVERos

\begin{abstract}
In this article are described dendritic structures containing photoactive groups at the surface or in the core. The observed supramolecular effects can be attributed to the nature of the photoactive group and their location in the dendritic architecture. The peripheric azobenzene groups in these dendrimeric compounds can be regarded as single residues that retain the spectroscopic and photochemical properties of free azobenzene moiety. The $E$ and $Z$ forms of higher generation dendrimer, functionalized with azobenzene groups, show different host ability towards eosin dye, suggesting the possibility of using such dendrimer in photocontrolled host-guest systems. The photophysical properties of many dendritic-bipyridine ruthenium complexes have been investigated. Particularly in aerated medium more intense emission and a longer excited-state lifetime are observed as compared to the parent unsubstituted bipyridine ruthenium complexes. These differences can be attributed to a shielding effect towards dioxygen quenching originated by the dendritic branches.
\end{abstract}

Key words: Supramolecular Photochemistry, Dendrimers, Azobenzene, Ruthenium Complexes.

\section{INTRODUCTION}

The combination of molecular components leads to supramolecular species and one of the most interesting aspects of the chemistry of supramolecular systems is their interaction with light (supramolecular photochemistry) (Balzani \& Scandola 1991). The assembly of components in a supramolecular system containing photoactive groups can produce two main effects (Fig. 1):

a) The individual components retain their intrinsic properties but the whole specie has new properties.

b) Some of the constituents of the assembled species have new properties.

In the first case there is no strong electronic interaction between the components of the molecular assembly, but in comparison with the individual components, a new microenviroment or steric

E-mail: gcazzell@quim.iq.usp.br modifications are created. In this class of systems, in general, a photocontrolled host-guest behavior is observed as in functionalized crown-ether (Shinkai et al. 1981) or cyclodextrin (Ueno et al. 1979) molecules. In the second one there is a significant electronic interaction between the assembled components or, alternatively, nuclear constraints that change the photophysical properties of the individual components are introduced, as in the case of many polynuclear (Bignozzi \& Scandola 1984) and caged (Pina et al. 1985) metal complexes.

In this short review supramolecular systems, based on dendritic structures containing photoactive groups will be described. The supramolecular effects, mentioned above, can be attributed to the nature of the photoactive groups and their location in the dendritic architecture.

\section{DENDRITIC SYSTEMS CONTAINING PERIPHERAL AZOBENZENE GROUPS}

Dendrimers (Newkome et al. 1996) are welldefined, highly branched macromolecules con- 
a)

b)

New Properties
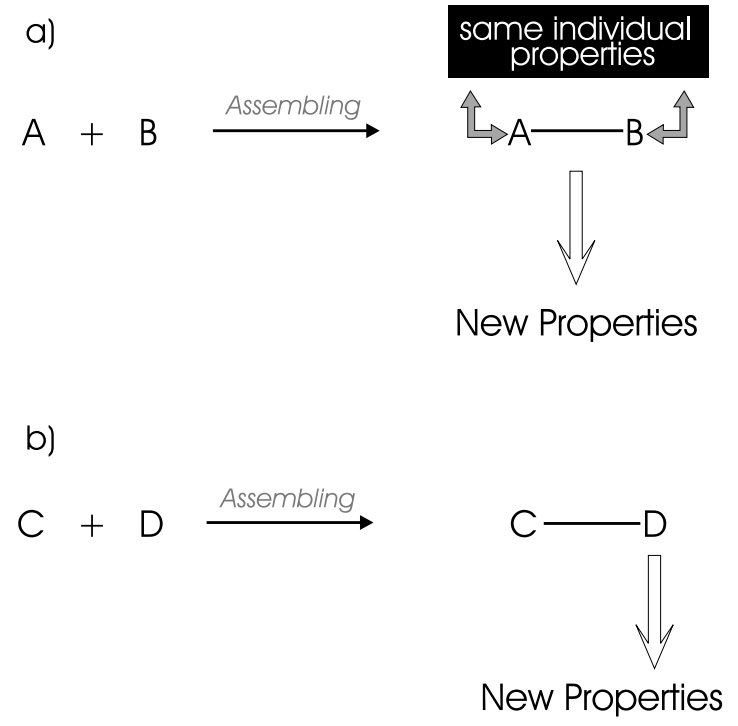

Fig. 1 - Supramolecular Effects: a) Week electronic/nuclear interaction leads to the maintenance of the individual properties; b) appreciable electronic/nuclear interactions leads to changes in the individual properties.

structed from an initiator core upon wich radially branched layers, termed generations, are covalently attached. As a result of the particular shape of dendritic molecules and the way branches propagate, three main regions can be defined in this class of compounds: a core, internal cavities and a surface. Recently, the preparation of two isomeric families of poly(propylene imine) dendrimers, containing at the surface para $(p)$ and meta $(m)$ carboxamidesubstituted azobenzene units as peripheral end groups, was described (Archut et al. 1998a). The total number of azobenzene residues for the first, second, third and fourth generations (G1 to G4) is respectively 4, 8, 16 and 32 . Fig. 2 shows the structure of the para-substituted G4 generation and the two para and meta model compounds, that will be simply called monomers.

All dendritic compounds exhibit the same absorption maximum for the $\pi-\pi^{*}$ and $n-\pi^{*}$ bands as for the corresponding monomer derivatives. The molar absortivities values, taking into account the experimental uncertainties, increase for each family according to an arithmetic progression, indicating that the overall molar absortivity corresponds to the addition of the molar absortivity of each single azobenzene chromophoric unit.

The $E$-azobenzene dendrimer units can be switched to the $Z$ form by light of appropriate wavelength and can be converted back to the $E$ form by either irradiation or by heating. Despite the decrease of quantum yields with increasing dendrimer generation, the quantum yield for each single photoactive unit is not dependent on the number of such units in the species.

The above mentioned spectroscopic and photochemical results indicate clearly that there is no strong interchromophoric interactions and/or effective steric hidrance in the surface region of such compounds. The azobenzene units in these dendrimeric compounds can be regarded as single residues that retain the spectroscopic and photochemical properties of the free azobenzene moiety.

In principle, the access of guest molecules to the internal cavities of the dendritic compounds can be controlled by surface isomerizable azobenzene groups, since there is a large structural rearrangement in going from the $E$ to the $Z$ isomer (Rau 1990).

Two distinct situations can be found, as represented schematically in (i), where the open and close forms of the surface correspond respectively to the linear $E$ and bent $Z$ isomers:

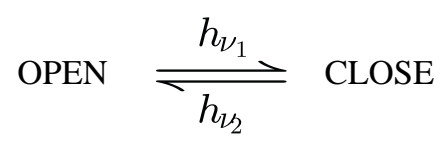

The host ability of these compounds towards eosin dye has been studied (Archut et al. 1998b). Both the $E$ and $Z$ forms of the dendrimers quench the eosin fluorescence by a static mechanism. The quenching is most likely due to an electron-transfer reaction between the singlet excited state of eosin and the tertiary amine units present along the branches of the dendrimers. Quenching by the $Z$ form is more efficient than quenching by the $E$ form. These results indicate that eosin is hosted by the dendrimers and suggest the possibility of using dendrimer derivatives in photocontrolled host-guest systems.

\section{METAL COMPLEXES CONTAINING DENDRITIC BIPYRIDINE LIGANDS}

The complexes of the $\left[\mathrm{Ru}(\mathrm{bpy})_{3}\right]^{2+}$ family (bpy= 2,2-bipyridine) show a unique combination of photophysical and redox properties (Juris et al. 1988). These properties can be significantly modified by the functionalization of the bpy ligand with dendrimeric arms (Issberner et al. 1997). The main effect is observed in the intrinsic photophysical prop- 


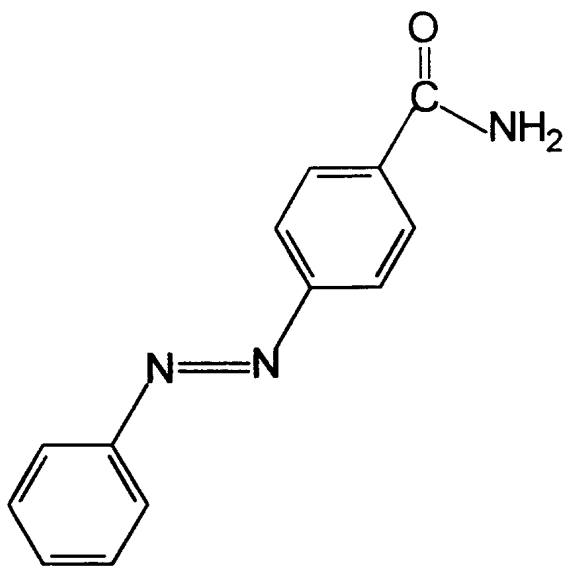

para-azobenzenecarboxylic acid amide

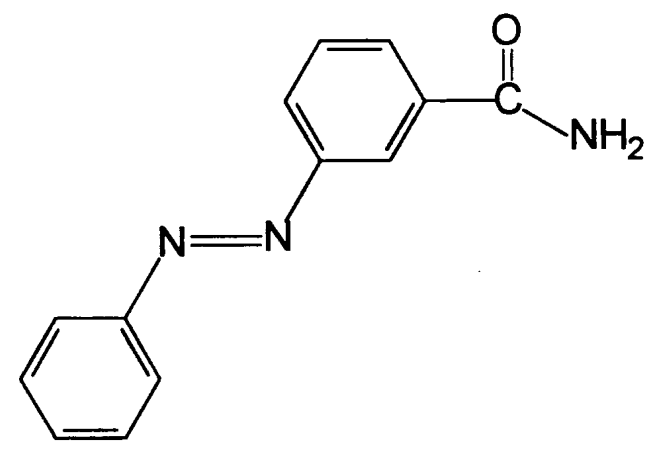

meta-azobenzenecarboxylic acid amide

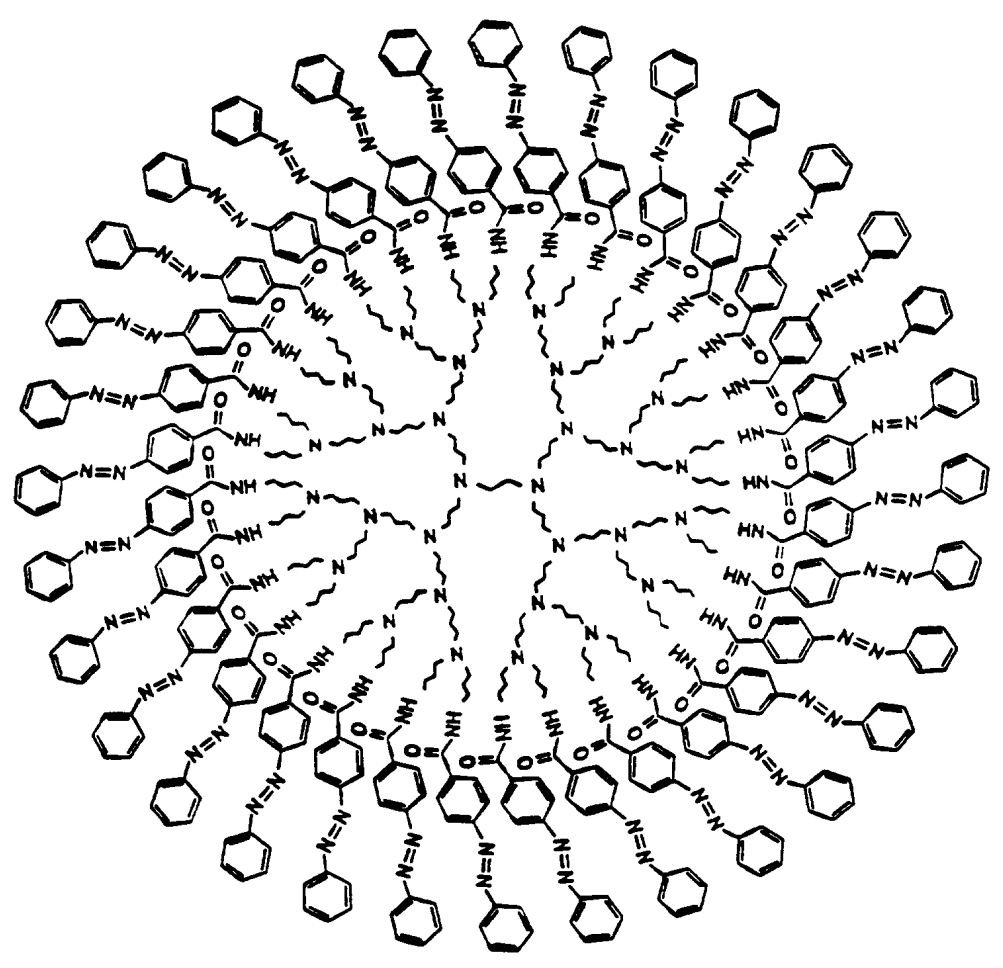

para-substituted fourth generation dendrimer (G4)

Fig. 2 - Structures of the monomeric model compounds and the G4 p-azobenzene substituted dendrimer. 


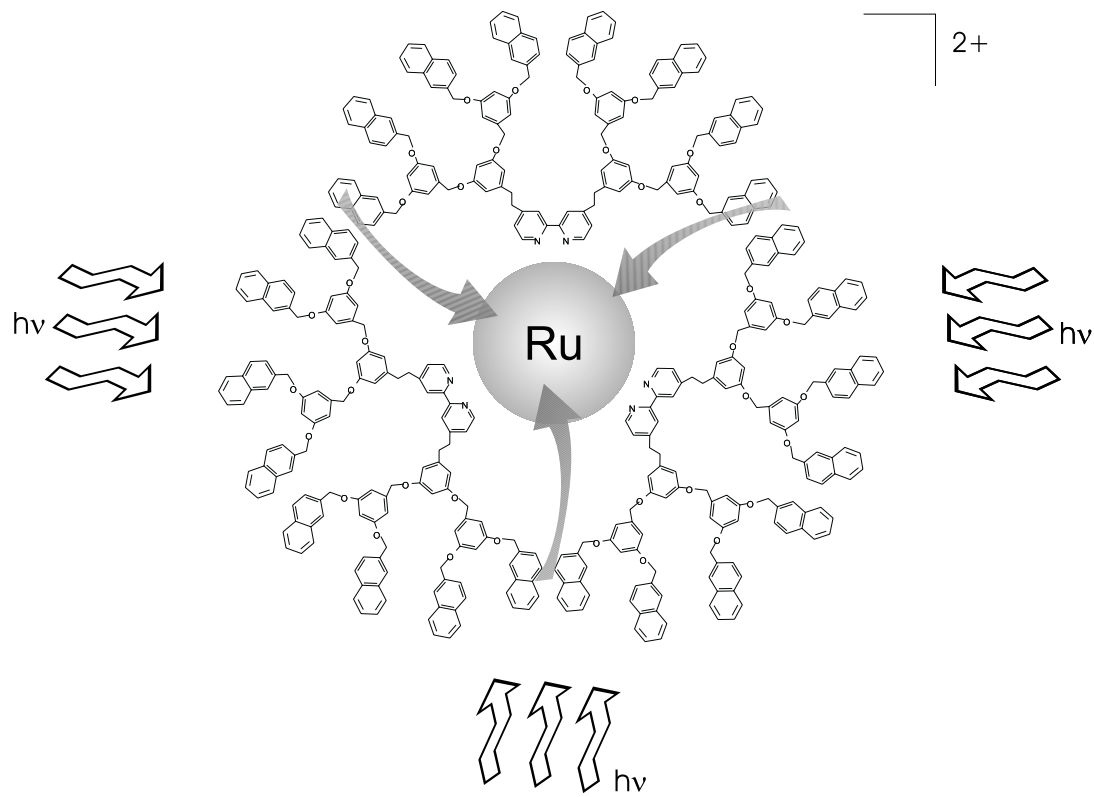

Fig. 3 - Schematic representation of ligth absorption and energy-transfer processes in the naphthalene substituted dedritic-bpy ruthenium(II) complex.

erties and in the excited state reactions with energy/electron transfer quenchers. In deaerated solution, higher generation bpy-dendrimer complexes exhibit a more intense emission and a longer excitedstate lifetime as compared to the parent unsubstituted $\left[\mathrm{Ru}(\mathrm{bpy})_{3}\right]^{2+}$ complex under the same conditions. The most interesting features are observed in aerated solution. The same trends in the photophysical properties are observed but in a remarkable way. For example, the excited-state lifetime of a ruthenium (II) complex containing 54 peripheral methyl esther groups in aerated and deaerated medium is respectively, 6 and 1.7 times greater than that for the parent unsubstituted $\left[\mathrm{Ru}(\mathrm{bpy})_{3}\right]^{2+}$. The differences can be attributed to a more rigid microenviroment at the core and to a shielding effect towards dioxygen quenching. A drastic decrease is also observed, in the bimolecular quenching rate constants, for the reactions of many bpy-dendritic ruthenium complexes and neutral, cationic and anionic electron-transfer quenchers (Vögtle et al. 1999). The above mentioned results show that the dendritic ligands are important as modulators of the central core behaviour.

Another interesting feature of this class of dendrimers, containing a central $\left[\mathrm{Ru}(\mathrm{bpy})_{3}\right]^{2+}$ unit, is the possibility of attaching, at the end of each branch, reactive groups such as energy donor species. A simple model for the photosyntetic antenna system (Gust 1990), was build up by coordination of naphthalene substituted dendritic-bpy to $\mathrm{Ru}^{2+}$ ions (Azzellini 1998). In this case, the reaction center and the energy collectors are represented, respectively, by the single $\left[\mathrm{Ru}(\mathrm{bpy})_{3}\right]^{2+}$ unit and naphthalene groups. The number of this energy donors is, as expected, dependent on each dendrimer generation. An efficient energy transfer process is achieved, when the samples are excited at the absorption band region of the aromatic units, as indicated by the quenching of naphthalene fluorescence (Plevoets et al. 1999), reproducing some aspects of the natural systems.

\section{REFERENCES}

Archut A, Azzellini GC, Balzani V, De Cola L \& Vögtle F 1998a. Azobenzene-Functionalized Cascade Molecules: Photoswitchable Supramolecular Systems. Chem Eur J 4: 669-706.

Archut A, Vögtle F, De Cola L, Azzellini GC, Balzani V, Ramanujam PS \& Berg RH 1998b. Towards Photoswitchable Dendritic Hosts. Interaction between Azobenzene- Functionalized Dendrimers and Eosin. J Am Chem Soc 120: 12187-12191. 
Azzellini GC, De Cola L, Balzani V, Issberner J, Ploenvets M \& Vögtle F 1998. Synthesis and Luminescence Properties of Dendrimeric Ruthenium(II) Complexes. XXXIII International Conference on Coordination Chemistry, Abstract. Book: $151 \mathrm{p}$.

Balzani V \& Scandola F 1991. In: Supramolecular Photochemistry, Ellis Horwood, Chichester.

Bignozzi CA \& Scandola F 1984. Cyano-Bridged Ruthenium(II)/Platinium(II) Complexes: Synthesis, Photophycal Properties and Excited-State Redox Behavior. Inorg Chem 23: 1540-1545.

Gust D \& More TA 1990. Photosynthetic Model Systems. Top Curr Chem 159: 103-152.
Issberner J, Vögtle F, De Cola L \& Balzani V 1997. Dendritic Bipyridine Ligands and Their Tris(bipyridine)Ruthenium(II) Chelates: Syntheses, Absorption Spectra, and Photophysical Properties. Chem Eur J 3: 706-712.

Juris A, Balzani V, Barigelletti F, Campagna S, Belser P \& von Zelewsky A 1988. Ru(II) Polypiridine Complexes: Photophysics, Photochemistry, Electrochemistry and Chemiluminescence. Coord Chem Rev 84: 85-277.

Newkome GR, Moorefield CN \& Vögtle F 1996. In: Dendritic Macromolecules: Concepts, Syntheses, Perspectives. VCH, Weinheim.

Pina F, Ciano M, Moggi L \& Balzani V 1985. Electron-Transfer Photosensitization via Ion-Pairs. Co(Sepulchrate $)^{3+}-$ Iodide Ion. Inorg Chem 24: 844-847.

Plevoets M, Vögtle F, De Cola L \& Balzani V 1999. Supramolecular Dendrimers with a $\left[\mathrm{Ru}(\mathrm{bpy})_{3}\right]^{2+}$ Core and Naphthyl Peripheral Units. New J Chem 23: 63-69.

Rau H 1990. Azo Compounds. In: Dürr H, BouasLaurent H. eds. Photochromism, Molecules and Systems. Amsterdam, Elsevier, p.165-192.

Shinkai S, Nakaji T, Ogawa T, Shigematsu K \& ManABE O 1981. Photocontrol of Ion Extraction and Ion Transport by a Bis(Crown-Ether) with a Butterfly like Motion. J Am Chem Soc 103: 111-115.

Ueno A, Yoshimura H, Saka R \& Osa T 1979. Photocontrol of Binding Ability of Capped Cyclodextrin. J Am Chem Soc 101: 2779-2780.

Vögtle F, Plevoets M, Nieger M, Azzellini GC, Credi A, De Cola L, De Marchis V, Venturi M \& BALZANi V 1999. Dendrimers with a Photoactive and redox-active $\left[\mathrm{Ru}(\mathrm{bpy})_{3}\right]^{2+}$-type core: Photophysical properties, electrochemical behaviour, and excited-state electron-transfer reactions. J Am Chem Soc 121: 6290-6298. 